\title{
Surface Resonance Bands on (001)W: Experimental Dispersion Relations
}

\author{
Willis, R. F.; Feuerbacher, B.; Christensen, N. Egede
}

Published in:

Physical Review Letters

Link to article, DOI:

10.1103/PhysRevLett.38.1087

Publication date:

1977

Document Version

Publisher's PDF, also known as Version of record

Link back to DTU Orbit

Citation $(A P A)$ :

Willis, R. F., Feuerbacher, B., \& Christensen, N. E. (1977). Surface Resonance Bands on (001)W: Experimental Dispersion Relations. Physical Review Letters, 38(19), 1087-1091. https://doi.org/10.1103/PhysRevLett.38.1087

\section{General rights}

Copyright and moral rights for the publications made accessible in the public portal are retained by the authors and/or other copyright owners and it is a condition of accessing publications that users recognise and abide by the legal requirements associated with these rights.

- Users may download and print one copy of any publication from the public portal for the purpose of private study or research.

- You may not further distribute the material or use it for any profit-making activity or commercial gain

- You may freely distribute the URL identifying the publication in the public portal 
manifestation of a threshold effect localized near $\theta_{i}=45^{\circ}$ is not unlikely. It has been suggested ${ }^{9}$ that singularities at threshold energies (vacuum branch points) should be detected as slope discontinuities in the $I-V$ curves. Feature $D$ (Fig. 3) shows a less definite correlation with the threshold condition $E_{T}-E_{0}=0$ than $B$ and $C$. The interpretation in terms of a true threshold effect, however, does not explain why two dips are observed. The physical nature of our observations remains somewhat unclear but those new results demonstrate the usefulness of high-resolution electrons spectrometry applied to diffraction experiments; it is hoped that they will stimulate other high-precision experimental studies and theoretical calculations about surface resonances and threshold effects.

The cooperation of Dr. J. P. Hobson, Dr. R. A. Armstrong, and Dr. E. V. Kornelsen, from the National Research Council of Canada, who provided the crystal and its electron heating gun, is greatly acknowledged; the authors are grateful to Dr. R. A. Armstrong for helpful discussions and to Dr. E. G. McRae from the Bell Laboratories, Murray Hill, N. J. for his permission to cite unpublished work (Ref. 17) he has communicated to us. This work has been supported by the National Research Council of Canada and le Mi- nistère de l'Education du Québec.

${ }^{1}$ P. J. Jennings and M. N. Read, Surf. Sci. $\underline{41}, 113$
$(1974)$.
${ }^{2}$ P. J. Jennings, Surf. Sci. $\underline{34}, 668(1973)$.
${ }^{3}$ E. G. McRae, Surf. Sci. $42,413,427(1974)$.
${ }^{4}$ P. J. Jennings, Surf. Sci. $\underline{25}, 513(1971)$.
${ }^{5}$ E. G. McRae, Surf. Sci. $\underline{25}, 491(1971)$.
${ }^{6}$ S. Sinharoy, R. M. Stern, and P. D. Goldstone, Surf. Sci. 30, 207 (1972).

${ }^{7}$ V. E. Heinrich, Surf. Sic. 49, 675 (1975).

${ }^{8}$ L. R. Bedell and H. E. Farnsworth, Surf. Sci. $\underline{41}$, 165 (1975).

${ }^{9}$ J. I. Gersten and E. G. McRae, Surf. Sci. $\underline{29}, 483$ (1972).

${ }^{10}$ D. Edwards and F. M. Propst, J. Chem. Phys. $\underline{56}$, 3184 (1972).

${ }^{11}$ P. J. Estrup and E. G. McRae, Surf. Sci. $\underline{25}, 1$

(1971).

${ }^{12}$ C. B. Duke and C. W. Tucker, Phys. Rev. Lett. $\underline{23}$, 1163 (1969).

${ }^{13}$ E. G. McRae and G. H. Wheatley, Surf. Sci. 29,342 (1972).

${ }^{14}$ A. Adnot, Y. Ballu, and J. D. Carette, J. Appl. Phys. $\underline{43}, 2796$ (1972).

${ }^{15}$ D. Roy, A. Delâge, and J. D. Carette, J. Phys. E $\underline{8}$, 109 (1975).

${ }^{16}$ B. J. Hopkins, G. D. Watts, and A. R. Jones, Surf. Sci. 52,715 (1975).

${ }^{17}$ E. G. McRae, J. M. Landwehr, and C. W. Caldwell, to be published.

\title{
Surface Resonance Bands on (001)W: Experimental Dispersion Relations
}

\author{
R. F. Willis and B. Feuerbacher \\ Space Science Department, European Space Research and Technology Center, Noordwijk, Holland \\ and \\ N. Egede Christensen \\ Physics Laboratory I, The Technical University of Denmark, 2800 Lyngby, Denmark \\ (Received 17 January 1977)
}

\begin{abstract}
A band of unbound surface states (resonances), located in an energy region above the vacuum threshold corresponding to an energy band gap in the electron states of the bulk crystal, has been observed by angle-resolved secondary-electron-emission spectroscopy. The experimental dispersion behavior is in agreement with the two-dimensional band structure of a clean (001)W surface recently proposed by Smith and Mattheiss.
\end{abstract}

The two-dimensional band structure for the prominent surface states/resonances associated with the clean (001)W surface, and that for a saturation coverage of chemisorbed $\mathrm{H}$ atoms, have recently been determined by a parametrized linear-combination-of-atomic-orbitals (LCAO) slabmodel calculation. ${ }^{1}$ The calculated energy versus parallel wave-vector ( $E$. vs $\overrightarrow{\mathrm{k}}_{\|}$) relations permit an identification of spectral features observed in angle-resolved photoemission measurements. ${ }^{2,3}$ In particular, the LCAO calculation predicts a band of unbound states located a few $\mathrm{eV}$ above the vacuum threshold, $E_{\text {vac }}$, which gives rise to structure appearing in the inelastic or secondary-electron part of the photoemission spectrum, ${ }^{3}$ the latter reflecting the electronic energy band struc- 


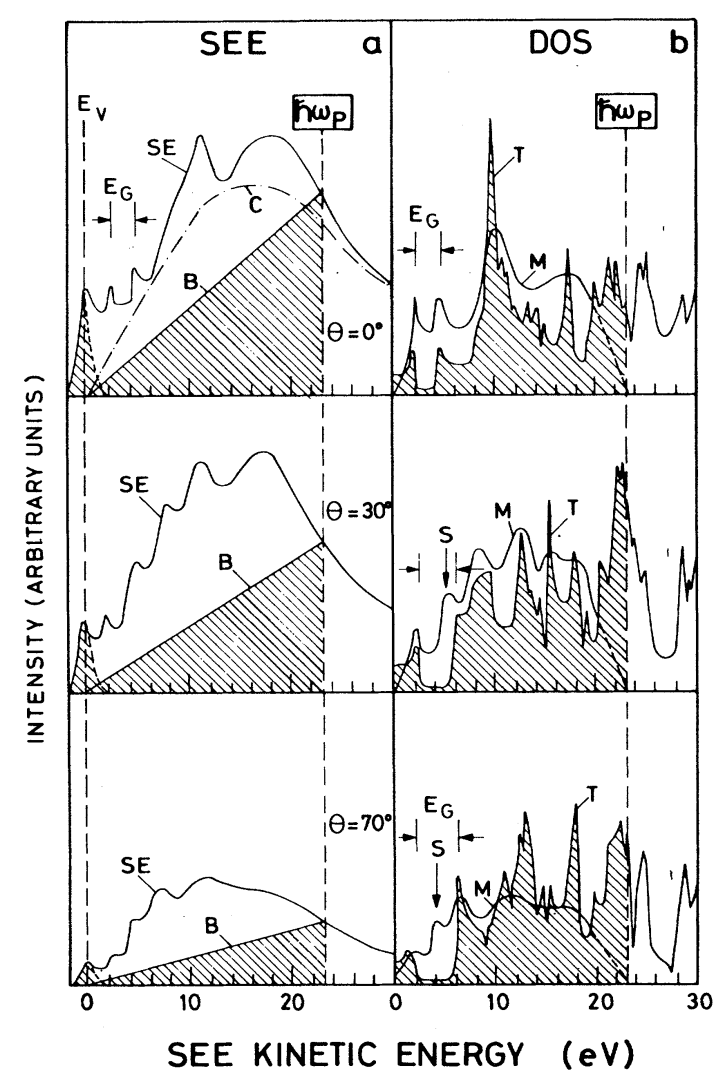

FIG. 1. Angle-resolved spectra of secondary electrons (SEE) emitted normal $\left(\theta=0^{\circ}\right)$ and in directions $\theta$ $=30^{\circ}$ and $\theta=70^{\circ}$ to a (001) $\mathrm{W}$ surface. The measured or "enhanced" density-of-states (DOS) profiles (curves $M$, column $b$ ) are compared with the theoretical DOS profiles (curves $T$, column b).

ture of the unbound states. We describe here the results of angle-resolved secondary-electron emission (SEE) spectroscopy on clean (001)W surfaces, which endorse this interpretation. Also, although effects due to unbound surface resonances have been reported previously in low-energy electron diffraction, ${ }^{4}$ as well as SEE spectroscopy, ${ }^{5}$ we believe that this work represents the first successful attempt to measure their complete dispersion relation on metal surfaces. The relationship to the bulk energy band structure is also clearly identified by this method.

Previous high-resolution SEE measurements, using a small angle selective cylindrical $130^{\circ}$ sector electrostatic analyzer ${ }^{6}$ have established that spectra (SE) measured normal to the lowindex (100), (110), and (111) faces of tungsten correlated closely with fine structure in the onedimensional density-of-states function along the corresponding symmetry directions in the bcc
Brillouin zone. ${ }^{7}$ Figure 1 (column $a$ ) shows the results obtained for $\langle 100\rangle$ symmetry-oriented emission normal to the (001)W face $\left(\theta=0^{\circ}\right)$ together with typical spectra obtained in off-symmetry directions, $\theta=30^{\circ}$ (middle panel) and $\theta=70^{\circ}$ (lower panel), the angle-resolved secondary electron current $j_{\mathrm{SE}}(E, \Omega)$ being defined for a series of emergent angles $\Omega$ in terms of the usual azimuthal angle $\theta$ and polar angle $\theta . \varphi$ was chosen such that the $\overrightarrow{\mathrm{k}}_{\|}$direction was scanned along a direction corresponding to $\bar{\Gamma} \bar{X}(\bar{\Delta})$ in the surface Brillouin zone. The angular variation was achieved by rotating the target crystal about an axis normal to the plane of incidence and reflection of a primary "exciting" electron beam, $E_{p} \simeq 100 \mathrm{eV}$. The changing angle of incidence of the impacting electron beam had no significant influence on the present results. Experimental details of the apparatus and crystal surface preparation technique have been described in previous publications. ${ }^{6}$

Bulk final-state fine-structure effects in SEE spectra are a consequence of a number of inelastic scattering processes which arise as a consequence of the dynamically screened Coulomb interaction between electrons in unbound states and the valence electron states of the crystal. Electron-hole pair, plasmon excitation and decay, and valence-hole Auger relaxation processes all play a role. However, the important point is that all mechanisms serve to populate unbound one-electron states, the number density of which factors out of an otherwise smooth background continuum distribution reflecting the convolution of all available filled (valence) and empty (conduction) singleparticle states. Inelastic "self-energy" damping effects will further serve to smear out this fine structure.

In the angle-integrated case, $\int_{0}^{\pi} j_{\mathrm{SE}}(E, \Omega) d \Omega$, this usually produces the familiar "cascade" distribution of inelastic electrons, bulk density-ofstates features appearing only weakly. ${ }^{8}$ This is not the case for the angle-resolved measurements, Fig. 1, and one immediate problem which arises is that no single "cascade" background (curve $C$ ) serves to fit the angle-dependent spectra from all three low-index faces. This is not unexpected when one considers that the initial- and finalstate distributions are very different for the different directions of emission and so might heavily weight the overall angle-dependent spectra from each low-index face. Clearly it is desirable, however, to be able to compare the SEE spectral fine structure with the bulk-states distribution curve free from this background inten- 
sity contribution. The subtraction of an arbitrary linear background function $B$ (together with a spurious instrumental peak), shaded (Fig. 1, column $a$ ), extending from the vacuum level $E_{\mathrm{kin}}=0$ $\mathrm{eV}$ to the dominant bulk plasmon threshold, $\hbar \omega_{p}$ $\simeq 24 \mathrm{eV}$, above which the single-particle fine structure becomes severely lifetime-broadened, permits comparison of the experimental numberdensity-of-unbound-states profile $(M)$ with the theoretical $(T)$ distribution. The density-of-states (DOS) calculations (column $b$, Fig. 1) have been performed in an extended-zone scheme so as to include elastic "umklapp" scattering effects from directions defined by up to seven shells of reciprocal lattice $G$ vectors emanating about a selected $\Gamma$ point. All relevant reduced-zone band-structure features are thus distinguished in the experimental spectra. ${ }^{9}$

As can be seen in Fig. 1, column $b$, the experimental results $(M)$ show good agreement with the envelope of the theoretical profile $(T)$, the latter assuming a constant Lorentzian broadening of only $0.25 \mathrm{eV}$. The apparent increased broadening, observed in curve $M$ up to the plasmon threshold $\hbar \omega_{p}$, is mainly due to an increasing number of contributing unbound states at points distributed throughout $\overrightarrow{\mathrm{k}}$ space at the higher energies. ${ }^{9}$ In particular, an energy band gap which extends throughout the Brillouin zone of tungsten centered approximately $9 \mathrm{eV}$ above the Fermi energy is clearly resolved in the experimental spectra as the feature $E_{G}$, Fig. 1. The angular variation of the energetic position and width of this spectral feature shows good agreement with the behavior predicted for emission from all three low-index faces by the bulk energy-band structure ${ }^{6,9}$ More importantly, its presence allows us to identify features which arise from conditions of enhanced surface sensitivity associated with "band-gap electron emission". ${ }^{10}$

In Fig. 1, all of the SEE spectral fine structure appearing in curve $M$ is explained in terms of bulk contributing states (curve $T)^{9}$ - except, that is, for the "anomalous" peak $S$ which moves into the band-gap region $E_{G}$ with increasing polar angle $\theta$. Measurements have been made in discrete $10^{\circ}$ steps over the range $0^{\circ} \lesssim \theta \lesssim 70^{\circ}$, showing the evolution of this feature into the bulk band gap, the spectra shown in Fig. 1 being representative. This feature $S$ is identified with emission due to an intrinsic surface resonance effect.

Surface states/resonances are described by evanescent wave functions with complex wave vectors having real $\overrightarrow{\mathrm{k}}_{\|}$but complex $\overrightarrow{\mathrm{k}}_{\perp}$ compo-

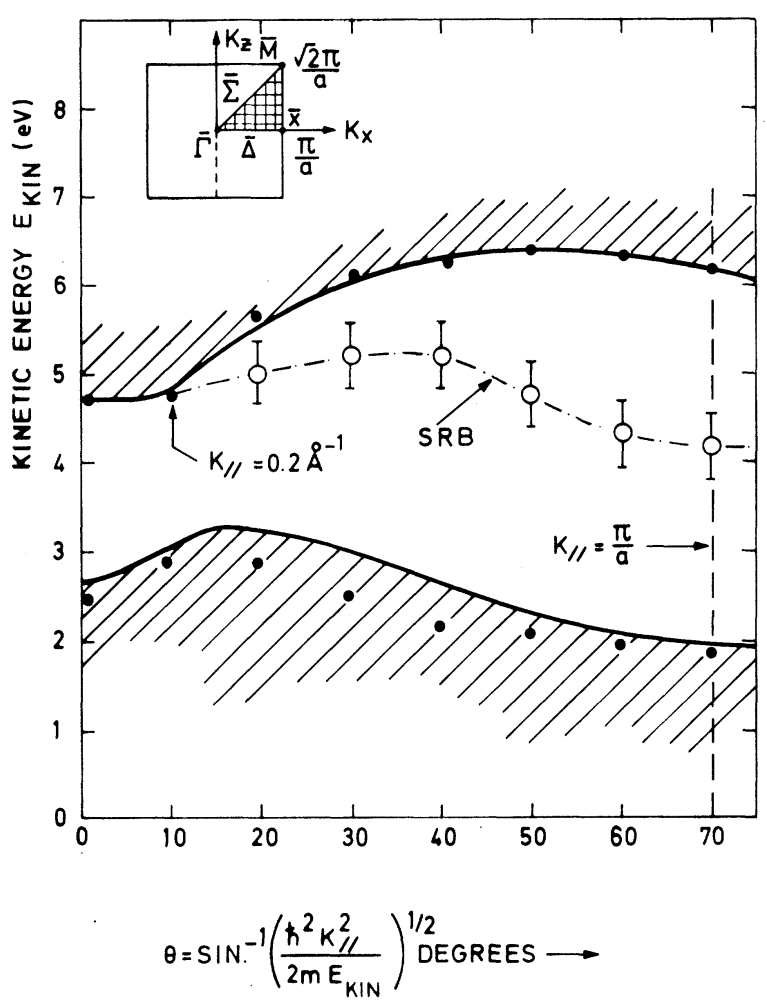

FIG. 2. Angular dependence of the (001) W band gap $E_{G}$ and the spectral peak $S$ (Fig. 1) as a function of polar angle $\theta$ : points, experiment; lines, theory, along the $\bar{\Delta}\langle 10\rangle$ direction of the surface Brillouin zone (inset).

nents. ${ }^{11}$ The energy eigenstates of the surface can be labeled by a two-dimensional wave vector $\overrightarrow{\mathrm{k}}_{\|}$which relates to the surface crystallography, i.e., in the usual way, one can confine the wave vectors $\overrightarrow{\mathrm{k}}_{\|}$associated with the evanescent Bloch functions to lie within the associated surface Brillouin zone. In Fig. 2, the energies of the (001)W upper and lower band-gap extremities $\left(E_{G}\right.$, Fig. 1) are plotted as a function of $\vec{k}_{\|}$, related to the polar angle $\theta$ by the usual free-electron relationship: $\theta=\sin ^{-1}\left\{\hbar^{2} \overrightarrow{\mathrm{k}}_{\|}{ }^{2} /\left(2 m E_{\mathrm{kin}}\right)\right\}^{1 / 2}$. Momentum normal to the surface $\vec{k}_{\perp}$ is not conserved so that for any particular value of $\vec{k}_{\|}$in the surface Brillouin zone (shown inset), the bulk bands along the surface normal direction give rise to the shaded regions indicated. ${ }^{12}$ The agreement between the theoretical (curves) and experimental (points) bulk band-gap behavior is seen to be better than $\pm 0.5 \mathrm{eV}$ over the whole angular range $0^{\circ} \lesssim \theta \lesssim 70^{\circ}$. A band of surface resonances can arise in the energy gap between the bulk states as a consequence of the periodic atomic lattice arrangement in the surface. The dispersion behavior of peak $S$ (Fig. 


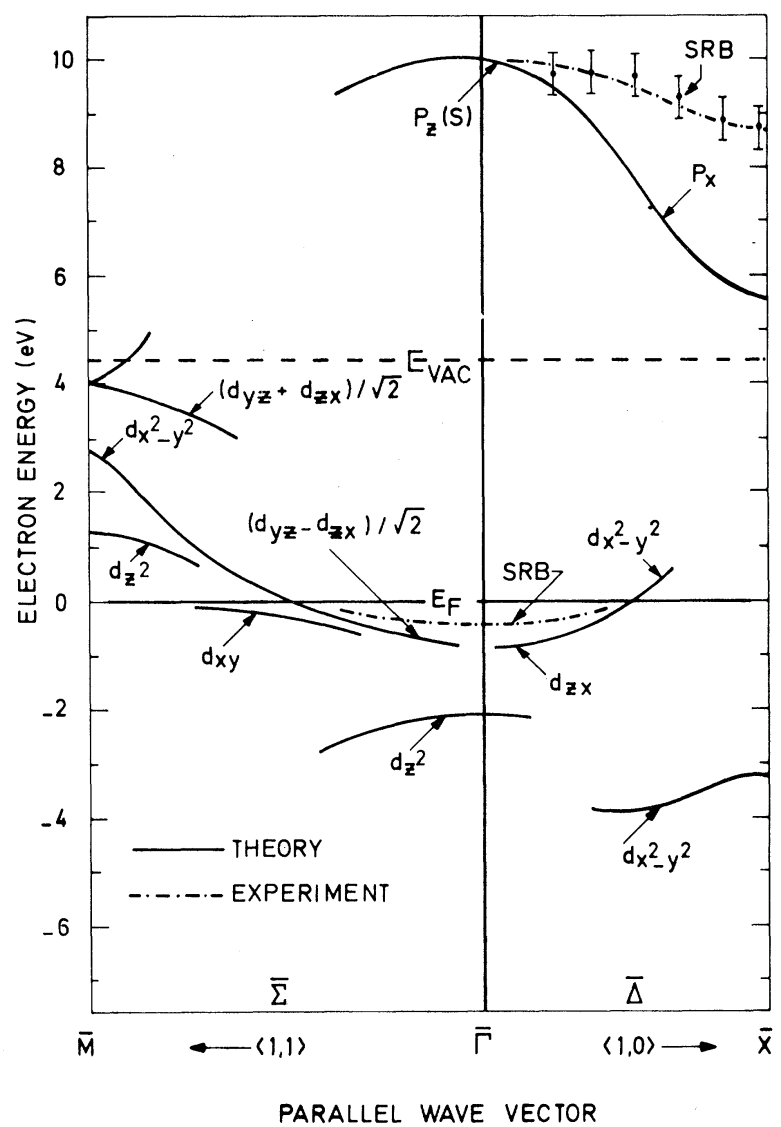

FIG. 3. Two-dimensional band structure of the clean (001)W surface: theory (Ref. 1), photoemission experiment (Ref. 2), and present SEE results (error bars indicated).

1) is plotted as a function of $\vec{k}_{\|}$along the surface Brillouin-zone symmetry direction $\bar{\Delta}$, the irreducible part of which is shown cross hatched (inset, Fig. 2). The point of interest in this case is that the dispersion behavior of the surface resonance band (SRB) is clearly indicative of the twodimensional surface Brillouin-zone boundary $\bar{X}$ at $\overrightarrow{\mathrm{k}}_{\|}=\pi / a \simeq 1 \AA$ for $\theta \simeq 70^{\circ}$. This result bears a strong resemblance to the theoretical dispersion relation predicted by the LCAO model calculations of Smith and Mattheiss. ${ }^{1}$

The two-dimensional energy bands for the clean (001)W surface along the two principal symmetry directions of the surface Brillouin zone, $\bar{\Delta}$ and $\bar{\Sigma}$, are displayed in Fig. 3. The present SEE results endorse the prediction of a band of unbound oneelectron states (SRB) existing above the vacuum level $E_{\text {vac }}$ in the appropriate energy range and with the correct sense of dispersion (downward in energy away from $\bar{\Gamma}$ ) along the $\bar{\Gamma} \bar{X}$ direction.
The LCAO model calculations identify those states/resonances whose eigenvector components are conspicuously large in the outermost atomic layer(s). Each of these states has been labeled with the $\mathrm{W}$ orbital which has the largest amplitude in the LCAO eigenvector, the "orbital content" of the states in many cases varying with position in $\vec{k}$ space. The above unbound SRB is seen to be derived from $\mathrm{W}(6 p)$ orbitals, the largest component of which changes from an admixture of $p_{z}(6 s)$ character close to $\bar{\Gamma}$, the $p_{x}$ component increasing and becoming dominant (as one might expect) at the larger polar angles. For the sake of completeness, the dispersion behavior of a band of bound one-electron states below the Fer$\mathrm{mi}$ level, which is observed in angle-resolved photoemission measurements, ${ }^{2}$ is shown also, Fig. 3. Again the sense of dispersion agrees with the theoretical predictions, although the absolute energies and shapes of both the experimental curves show some disagreement. This is not too surprising in view of the fact that theoretical curves are extremely sensitive to the particular choice of LCAO parameters. ${ }^{1}$ This being the case, the agreement with experiment is remarkably good.

In summary, the present results provide an incisive test of the LCAO treatment ${ }^{1}$ of the origin of the resonances observed on the (100) surfaces of $\mathrm{W}\left(\right.$ and $\mathrm{Mo}^{13}$ ). The observations relate to the nature and variation with position in $\vec{k}$ space of the atomic orbital content of the $\overrightarrow{\mathrm{k}}_{\|}$-resolved surface local density-of-states distribution. Strong emission occurs from those states coincident with gaps in the bulk energy-band structure. These general conclusions have been endorsed by experimental results from quite different experiments: angle-resolved photoemission, ${ }^{2}$ fieldemission, ${ }^{13}$ and now, secondary-electron emission spectroscopy.

The authors are indebted to M. R. Barnes for technical assistance and to Dr. B. Fitton for support. We are grateful also to Dr. N. V. Smith and Dr. L. F. Mattheiss for furnishing us with numerical data for Fig. 3. One of us (N.E.C.) thanks the Danish National Science Research Council for partial support.

\footnotetext{
${ }^{1}$ N. V. Smith and L. F. Mattheiss, Phys. Rev. Lett. 37, 1494 (1976).

${ }^{2}$ W. F. Egelhoff and D. L. Perry, Phys. Rev. Lett. 34, 93 (1975); R. F. Willis, B. Feuerbacher, and B. Fitton, Solid State Commun. 18, 1315 (1976).

${ }^{3}$ B. Feuerbacher and R. F. Willis, Phys. Rev. Lett.
} 
36,1339 (1976).

${ }^{4}$ E. G. McRae, Surf. Sci. 25, 491 (1971); J. Lauzier, L. de Bersuder, and V. Hoffstein, Phys. Rev. Lett. 27, 735 (1971).

${ }^{5}$ P. E. Best, Phys. Rev. Lett. 34, 674 (1975); R. F. Willis, Faraday Discuss. Chem. Soc. 60,245 (1975).

${ }^{6}$ R. F. Willis, Phys. Rev. Lett. 34, 670 (1975).

${ }^{7}$ Measurements analogous to the angle-resolved photoemission results of N. E. Christensen and B. Feuerbacher, Phys. Rev. B 10, 2349, 2373 (1974).

${ }^{8}$ E. J. Scheibner and L. N. Tharp, Surf. Sci. 8,247 (1967).

${ }^{9}$ R. F. Willis and N. E. Christensen, in Proceedings of the International Symposium onPhotoemission, Noordwijk, The Netherlands, 1976, edited by R. F. Willis et al., publication no. SP118 (European Space Research Organization, Paris, 1976). The intensity of the "background emission," particularly that beneath the bandgap region $E_{G}$ (Fig. 1), can arise from several sources, including "surface emission" from atoms in the outermost atomic layer emitting directly into vacuum.

${ }^{10}$ P. J. Feibelman and D. E. Eastman, Phys. Rev. B 10,4932 (1974).

${ }^{11}$ V. Heine, Proc. Phys. Soc., London 81, 300 (1962).

${ }^{12}$ M. Henzler, Surf. Sci. 25, 650 (1971).

${ }^{13}$ S.-L. Weng, Phys. Rev. Lett. $\underline{38}, 434$ (1977).

\title{
Measurement of Microwave-Enhanced Energy Gap in Superconducting Aluminum by Tunneling*
}

\author{
Tom Kommers and John Clarke \\ Department of Physics, University of California, and Materials and Molecular Research Division, \\ Lawrence Berkeley Laboratory, Berkeley, California 94720
}

(Received 7 February 1977)

\begin{abstract}
$\mathrm{Al}-\mathrm{Al}_{2} \mathrm{O}_{3}-\mathrm{Al}$ tunnel junctions were used to measure large increases in the energy gap of superconducting aluminum films in the presence of $10-\mathrm{GHz}$ microwave radiation. When the microwave power was increased at constant temperature, enchancement occurred only for temperatures at which twice the equilibrium energy gap exceeded the photon energy. When the temperature was increased at constant microwave power, enhancement was observed at higher temperatures.
\end{abstract}

Eliashberg and co-worker $\mathrm{s}^{1}$ have predicted that the energy gap, $\Delta$, of a superconducting thin film may be enhanced by microwave irradiation. In their model, photons of frequency $\nu<2 \Delta / h$ excite quasiparticles from states near the bottom of the excitation spectrum to states of higher energy. Thus, additional pair states near the Fermi wave vector, $k_{\mathrm{F}}$, become available for occupancy. Since the pair states near $k_{\mathrm{F}}$ contribute most strongly to the pairing interaction, this redistribution of pair-state occupancy increases the condensation energy, and leads to an increase in $\Delta$. Eliashberg and co-workers ${ }^{1}$ have suggested that this increase would account for the microwave enhancement of the critical current of superconducting microbridges. ${ }^{2}$ Subsequently, several experiments have supported the concept of gap enhancement: phonon-induced enhancement of the critical current of superconducting microbridges and point contacts, ${ }^{3}$ microwave-induced enhancement of the critical currents and transition temperatures of aluminum strips, ${ }^{4}$ and $\mathrm{mi}-$ crowave-induced enhancement of the voltage at which gap structure occurs in superconducting point contacts. ${ }^{5}$ Recently, Chang and Scalapino ${ }^{6}$ have performed detailed computer calculations of gap enhancement in which the energy dependence of the quasiparticle recombination time, and the effects of the nonequilibrium phonon distribution are included.

We have used $\mathrm{Al}-\mathrm{Al}_{2} \mathrm{O}_{3}-\mathrm{Al}$ tunnel junctions to measure large increases in the energy gap of superconducting aluminum films in the presence of $X$-band radiation. ${ }^{7}$ We define $\left\langle\Delta_{e}\right\rangle$ as the average equilibrium gap for the two films of a given junction, and $T_{\nu}$ as the temperature at which $h \nu$ $=2\left\langle\Delta_{e}\right\rangle$. When the microwave power was increased from zero with the junction at fixed temperature, $T,\langle\Delta\rangle$ increased for $T<T_{\nu}$, and decreased for $T \gtrsim T_{\nu}$. Alternatively, when we maintained constant microwave power and increased the temperature from below $T_{\nu}$, gap enhancement was observed to a temperature $T_{\downarrow}$, where $T_{\downarrow}>T_{\nu} \cdot T_{\downarrow}$ increased with microwave power. When the temperature was subsequently lowered at constant microwave power, the gap abruptly reappeared at a temperature significantly lower than $T_{\downarrow}$.

The $\mathrm{Al}-\mathrm{Al}_{2} \mathrm{O}_{3}-\mathrm{Al}$ junctions were fabricated in a cross-film geometry with a film width of about $300 \mu \mathrm{m}$, and film thicknesses in the range 80 to $300 \mathrm{~nm}$. Normal-state resistances ranged from 1 to $10 \Omega$. The transition temperatures of the 